\title{
Wide-range optical spin orientation in Ge from near-infrared to visible light
}

\author{
C. Rinaldi, ${ }^{*}$ M. Cantoni, M. Marangoni, C. Manzoni, G. Cerullo, and R. Bertacco \\ Dipartimento di Fisica, Politecnico di Milano, Piazza Leonardo da Vinci 32, 20133 Milano, Italy \\ (Received 30 December 2013; revised manuscript received 2 September 2014; published 16 October 2014)
}

\begin{abstract}
Ge-based spin-photodiodes have been employed to investigate the spectral dependence of optical spin orientation in germanium, in the range $400-1550 \mathrm{~nm}$. We found the expected maximum in the spin polarization of photocarriers for excitation at the direct gap in $\Gamma(1550 \mathrm{~nm})$ and a second sizable peak due to photogeneration in the $L$ valleys $(530 \mathrm{~nm})$. Data suggest distinct spin depolarization mechanisms for excitation at $\Gamma$ and $L$, with shorter spin relaxation times whether the $X$ point is involved. These devices can be used as integrated photon-helicity detectors over a wide spectral range.
\end{abstract}

DOI: 10.1103/PhysRevB.90.161304

PACS number(s): 72.25.Dc, 73.40.Qv, 85.60.-q, 85.75.-d

Spin-optoelectronics is a novel branch of semiconductor spintronics, aiming at adding a new degree of freedom to optoelectronics: the photon helicity. Exploiting the interplay between the photon angular momentum and the spin of electrons, integrated emitters (spin-LEDs) and detectors (spin photodiodes) of circularly polarized light have been proposed. Although GaAs is traditionally the material of choice for spin optoelectronics, because of its direct gap allowing for efficient conversion between light and spin polarization, recently $\mathrm{Ge}$ has attracted a considerable attention. In fact, thanks to the inversion symmetry of the crystal and the related absence of the $\mathrm{D}^{\prime}$ Yakonov-Perel spin scattering mechanism, Ge presents a longer spin coherence time than the one of GaAs. Spin manipulation [1], spin transport [2], spin optical pumping in the infrared [3-7], and electrical spin injection [8] in Ge have been reported. In our previous works $[9,10]$, we demonstrated the room temperature operation of spin-PDs based on fully epitaxial $\mathrm{Fe} / \mathrm{MgO} / \mathrm{Ge}(001)$ heterostructures, working at $0.95 \mathrm{eV}[11,12]$. However, there is still a poor understanding of electrons and holes optical spin orientation in $\mathrm{Ge}$, especially at photon energies much higher than the direct gap $(0.8 \mathrm{eV})$. The optical spin orientation in Ge over a wide spectral range has been theoretically investigated by Rioux and Sipe [13]. However, no experimental data for excitation far from the $\Gamma$ point of the Brillouin zone (BZ) have been reported so far. Even for GaAs, only very recently the photon energy dependence of optical spin orientation well above the absorption edge has been reported [14].

In this Rapid Communication, we report on the spectral dependence of the optical spin orientation in $\mathrm{Ge}$ at room temperature and over a wide spectral range $(400-1550 \mathrm{~nm}$, corresponding to $3.1-0.8 \mathrm{eV}$ ), via measurements of the helicitydependent photocurrent on Ge-based spin PDs. Surprisingly enough, the maximum sensitivity to photon helicity is observed far away from the direct gap $(0.8 \mathrm{eV})$, where optical pumping is supposed to produce the highest initial spin polarization. Indeed, the helicity-dependent photocurrent variation indicates a first peak around $1500 \mathrm{~nm}(0.8 \mathrm{eV})$ and then an absolute maximum of about $10 \%$ at $\sim 530 \mathrm{~nm}(\sim 2.3 \mathrm{eV})$, corresponding to optical pumping in the $L$ valley of the Ge band structure,

*christian.rinaldi@polimi.it as shown in Fig. 1(a). Fitting of our data within a simple diffusive model allows to gain a deeper insight into the physics of spin depolarization phenomena occurring when pumping at different photon energies. Different equivalent spin diffusion lengths must be taken into account when photocarriers are optically generated in the $\Gamma$ and $L$ valleys, respectively, thus suggesting that spin depolarization phenomena during the $\Gamma-X-L$ transitions play a major role.

$\mathrm{Fe} / \mathrm{MgO} / \mathrm{Ge}$ heterostructures have been prepared by molecular beam epitaxy $[11,15]$. We employed lightly $n$-doped Ge substrates $\left(\rho_{\mathrm{sc}} \sim 47 \Omega \mathrm{cm}\right)$ to maximize the spin diffusion length. Spin-PDs with circular shape and different areas have been fabricated via optical lithography and ion beam etching.

A sketch of a spin photodiode is shown in Fig. 1(b) [9]. In our experiments a laser beam with tunable wavelength, from 400 to $1550 \mathrm{~nm}$, impinges perpendicularly on the photodiode surface. The light helicity is modulated between right $(\sigma+)$ and left $(\sigma-)$ circular polarization, by means of a photoelastic modulator operating at a frequency of $50 \mathrm{kHz}$. The helicitydependent photocurrent flowing across the photodiode $(\Delta I=$ $\left.I^{\sigma+}-I^{\sigma-}\right)$ is measured using a lock-in amplifier, while the magnetization of the Fe layer is driven out-of-plane, parallel or antiparallel to the photon angular momentum, by means of an electromagnet. $\Delta I$ can be expressed as [9]

$$
\Delta I\left(V_{\text {bias }}\right)=2 \sigma_{ \pm} I_{\text {photo }}\left(D+A_{\mathrm{SF}}\right)
$$

where $I_{\text {photo }}\left(V_{\text {bias }}\right)$ is the unpolarized photocurrent measured for incident linearly polarized light and in-plane magnetization, $D$ is the Magnetic Circular Dichroism (MCD) due to dichroic absorption from the Fe layer (see Ref. [16] for details on its measurements and subtraction from raw data), and $A_{\mathrm{SF}}$ is the spin dependent transport asymmetry. The last term includes: (i) the degree of initial spin polarization after spin optical pumping, (ii) the effect of spin depolarization during transport towards the barrier, and (iii) the spin dependent transmission through the tunneling barrier. In this sense, $A_{\mathrm{SF}}$ is the key parameter for discussing the spectral dependence of spin optical orientation.

Figure 2(a) shows the experimental photocurrent $I_{\text {photo }}$ versus the applied bias $\left(V_{\text {bias }}\right)$ in case of a spin PD with 1.8-nm-thick $\mathrm{MgO}$ and several values of the photon energy. For each value of $h v$, we regulated the source intensity in order to obtain the same photocurrent for zero applied bias, ensuring the same exciton generation rate. In the working 

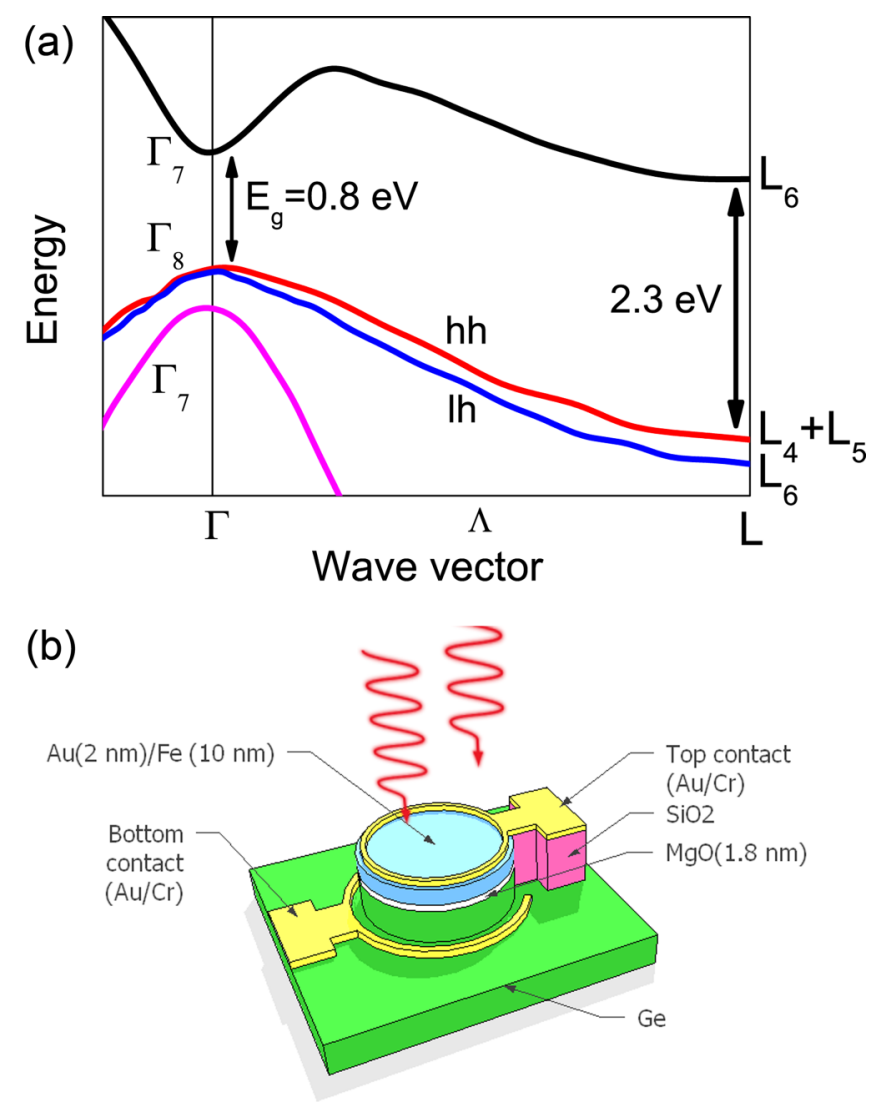

FIG. 1. (Color online) (a) Band structure of Ge from the $\Gamma$ to the $L$ point of the Brillouin zone (along the direction $\Lambda$ ). (b) Sketch of a $\mathrm{Fe} / \mathrm{MgO} / \mathrm{Ge}$ spin photodiode.

range of our $\mathrm{Fe} / \mathrm{MgO} / \mathrm{Ge}$ photodiodes, $-0.4 /+0.4 \mathrm{~V}, I_{\text {photo }}$ presents the typical $\mathrm{s}$ shape expected for photodetectors, without any sizable dependence on the photon energy. The photocurrent is null when $V_{\text {bias }}$ approaches the built-in voltage $V_{\text {bi }}$ [9], and tends to saturate when $\left|V_{\text {bias }}\right|$ increases, indicating that the photocurrent is limited only by the photogeneration process and basically all the excited carriers are collected at the electrodes at $\pm 0.4 \mathrm{~V}$ [17]. Figure 2(b) shows the experimental behavior of the helicity-dependent photocurrent $\Delta I\left(V_{\text {bias }}\right)$ for the same set of photon energies. Since for positive (negative) bias the electric field in the depletion region drives photogenerated electrons (holes) towards the $\mathrm{MgO}$ barriers, the modulation of $\Delta I$ arises from spin filtering of electrons (holes) in forward (reverse) bias. The corresponding spin transport asymmetry $A_{\mathrm{SF}}$ is plotted in Fig. 2(b) after subtraction of the MCD contribution [16]. Apart from the modulation of $A_{\mathrm{SF}}$ as a function of the photon energy discussed below, the shape of the photocurrent $I_{\text {photo }}$ versus bias, as well as that of $\Delta I$ and $A_{\mathrm{SF}}$, is almost independent on the photon energy, thus suggesting a similar transport mechanism across the barrier for all the photon energies employed. With reference to the Ge band structure sketched in Fig. 1(a), for photons resonant with the direct gap in $\Gamma$, photogenerated holes stay in $\Gamma$, while electrons suffer from a fast decay to the absolute conduction band minimum (CBM) in $L$, with a relaxation time $\tau_{\Gamma \rightarrow L} \sim 230$ fs [18]. At higher photon energy, instead, carriers can be directly generated in $L$ and

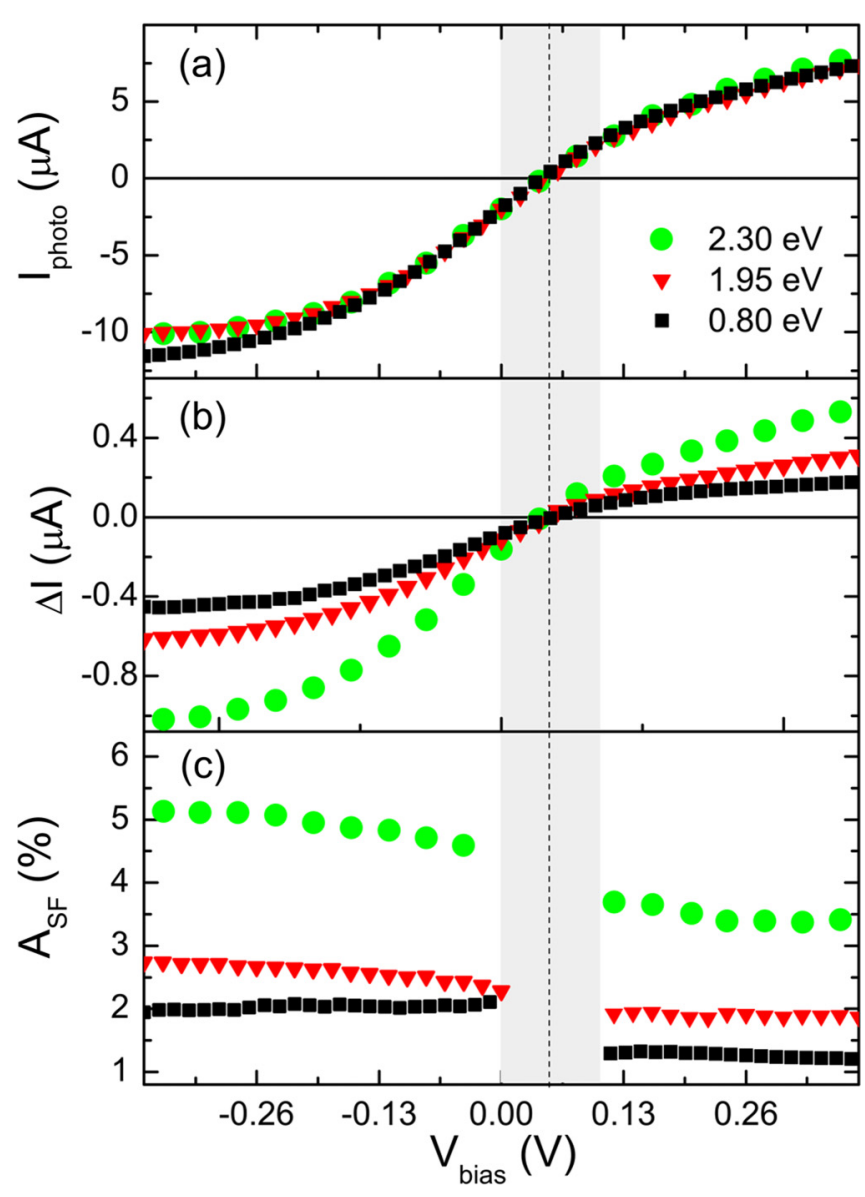

FIG. 2. (Color online) (a) Photocurrent $I_{\text {photo }}$ photogenerated by linearly polarized light vs the bias voltage $V_{\text {bias }}$, for several values of the photon energy $h v$. (b) Helicity-dependent photocurrent $\Delta I$ due to the full reversal of the circular polarization of light. (c) Spin transport asymmetry $\left(A_{\mathrm{SF}}\right)$ after subtraction of the MCD contribution. Data are not shown in the region were $\Delta I$ is almost zero and $A_{\mathrm{SF}}$ diverges [shaded region of (c)].

the situation is the opposite: electrons stay in $L$, while holes decay towards the valence band maximum (VBM) in $\Gamma$. Also for holes, however, the intervalley relaxation process was found to be very fast ( $\tau_{L \rightarrow \Gamma} \sim 200$ fs according to Ref. [4]). Noteworthy, these characteristic relaxation times are shorter than the average transit time $\tau_{t r}$ of photocarriers from the point of generation to the barrier (see Ref. [16]). Overall, we can assume that transport in the semiconductor and tunneling through the barrier take place always in $L$ and $\Gamma$ for electrons and holes, respectively, and points towards a minor role of hot carriers in our devices. This indicates that the energy dependence of the barrier transmission does not contribute to the observed dependence of $A_{\mathrm{SF}}$ on $h v$ and this is coherent with the very similar shapes of $I_{\text {photo }}, \Delta I$, and $A_{\mathrm{SF}}$ for all $h v$ (see Fig. 2).

Figure 3(a) reports $A_{\mathrm{SF}}$ values measured from $0.8 \mathrm{eV}$ to $3.1 \mathrm{eV}$ photon energy, both for holes $\left(V_{\text {bias }}=-0.4 \mathrm{~V}\right.$, red empty dots) and electrons ( $V_{\text {bias }}=+0.4 \mathrm{~V}$, blue filled dots) and for the magnetization of the iron layer saturated in the outof-plane direction (see Ref. [16]). Noteworthy, $A_{\mathrm{SF}}$ is slightly higher for holes than for electrons in all the investigated photon 


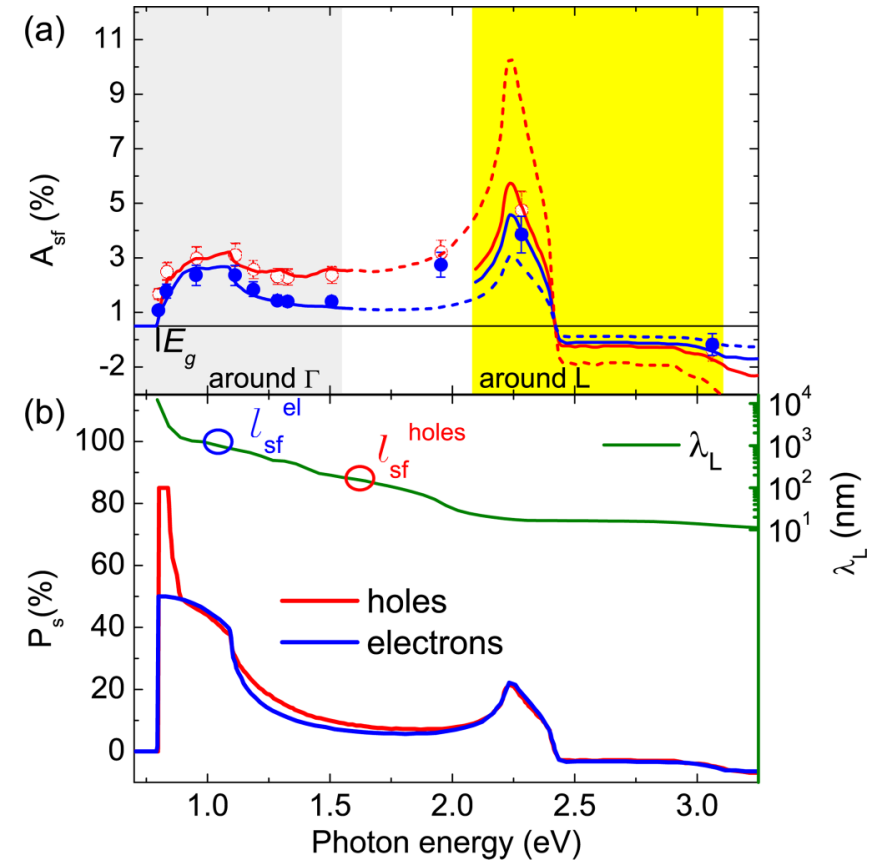

FIG. 3. (Color online) Spin filtering asymmetry of spin PD's vs photon energy. Continuous and dashed lines represent fits obtained with the diffusive model presented in the text. (b) Calculated degree of optical spin orientation $P_{S}$ [13]. The light absorption length $\lambda_{L}$ [19] is compared with the spin-diffusion length $l_{\mathrm{SF}}$ values for carriers [9] excited around $\Gamma$ (open circles on the $\lambda_{L}$ curve).

energy range, confirming our previous suggestion [9] of a sizable equivalent spin diffusion length for holes. Even more interestingly, Fig. 3(a) shows a nonmonotonic evolution of $A_{\mathrm{SF}}$ versus photon energy, which allows to gain a deeper insight into the process of spin optical pumping. Surprisingly, $A_{\mathrm{SF}}$ presents a relative maximum at about $1 \mathrm{eV}$, slightly above the absorption edge of $\mathrm{Ge}(\sim 0.8 \mathrm{eV})$, but then it reaches the absolute maximum at $\sim 2.3 \mathrm{eV}$, before assuming small negative values above $2.4 \mathrm{eV}$. This is unexpected, because the maximum degree of optical spin orientation should be achieved at the direct band gap of the semiconductor, according to $k \cdot p$ calculations [13].

In order to shed light on the apparent contradiction between the calculated degree of optical spin orientation and the measured $A_{\mathrm{SF}}$, we use a simple diffusive model of spin-PDs including spin-optical pumping [9]. It represents only a firstorder approximation for a semiconductor such as Ge, but contains the essential physics to describe the spectral response of our devices. The spin filtering asymmetry $A_{\mathrm{SF}}$ is given by [16]

$$
A_{\mathrm{SF}}=P_{S} \frac{l_{\mathrm{SF}}}{\lambda_{L}+l_{\mathrm{SF}}}\left[\frac{\gamma r_{B} r_{\mathrm{sc}}}{R_{\mathrm{sc}}\left(r_{\mathrm{sc}}+r_{B}\right)+\left(1-\gamma^{2}\right) r_{B}{ }^{2}+r_{B} r_{\mathrm{sc}}}\right],
$$

where $P_{S}$ is the carriers spin polarization immediately after the photogeneration [13]; $\lambda_{L}$ is the light absorption length; $\gamma$ is the spin-dependent interfacial resistance asymmetry; $r_{B}$ is the resistance per unit surface of the tunneling barrier; $R_{\mathrm{sc}}=$ $\rho_{\mathrm{sc}} L$ is the product between the semiconductor's resistivity
( $\left.\rho_{\mathrm{sc}}\right)$ and the Ge thickness $(L) ; r_{\mathrm{sc}}=\rho_{\mathrm{sc}} l_{\mathrm{SF}}$ is the product between $\rho_{\mathrm{sc}}$ and the equivalent spin diffusion length $\left(l_{\mathrm{SF}}\right)$ in the semiconductor of the carriers involved in tunneling. $l_{\mathrm{SF}}$ is defined as $l_{\mathrm{SF}}=\sqrt{D \tau_{\mathrm{SF}}}$, where $D$ is the diffusion coefficient and $\tau_{\mathrm{SF}}$ the spin-flip time of the carriers in Ge. In Eq. (2), the dependence on the photon energy $h v$ mainly arises from the term $f(h v)=P_{S}\left(\frac{l_{\mathrm{SF}}}{l_{\mathrm{SF}}+\lambda_{L}}\right)$ via $P_{S}$ and $\lambda_{L}$, which strongly depend on $h v$ as well as via the possible dependence of $l_{\mathrm{SF}}$ on the peculiar point of the BZ where carriers are generated for different photon energies.

Equation (2) allows to distinguish two operating regimes for spin-PDs, corresponding to the following expressions for $A_{\mathrm{SF}}$ :

$$
A_{\mathrm{SF}}=\left\{\begin{array}{ll}
P_{S} \frac{l_{\mathrm{SF}}}{\lambda_{L}} & \text { for } \lambda_{L} \gg l_{\mathrm{SF}} \\
P_{S} & \text { for } \lambda_{L} \ll l_{\mathrm{SF}}
\end{array} .\right.
$$

For $\lambda_{L} \gg l_{\mathrm{SF}}$, in the regime that we call spin depolarization regime, carriers are photogenerated in a deep layer and suffer from a major depolarization during their motion towards the barrier, so that $A_{\mathrm{SF}}$ is strongly suppressed. On the contrary, if $\lambda_{L} \ll l_{\mathrm{SF}}$, in the true optical spin-orientation regime, carriers are photogenerated in a layer much thinner than the spin diffusion length, so that they preserve their initial spin polarization till they reach the tunneling barrier. Figure 3(b) reports the measured values of $\lambda_{L}$ and the calculated values of $P_{S}$ versus photon energy, taken from Refs. [19] and [13] respectively. In the same figure, the $l_{\mathrm{SF}}$ values for holes and electrons, from our previous work on slightly doped $n$-Ge considering excitation close to the direct gap [9], are marked with circles on the $\lambda_{L}$ curve $(1 \mu \mathrm{m}$ for electrons and $150-220 \mathrm{~nm}$ for holes). It turns out that the spin depolarization regime, where $\lambda_{L} \gg l_{\mathrm{SF}}$, corresponds to photon energies close to the band gap of the semiconductor. Here, $\lambda_{L}(\sim 12.5 \mu \mathrm{m}$ at $0.8 \mathrm{eV}$ [19]) is very large with respect to the $l_{\mathrm{SF}}$ values. Noteworthy, in this energy range, $\lambda_{L}$ steeply increases when the photon energy decreases. As a result, $A_{\mathrm{SF}}$ decreases when moving from 1 to $0.8 \mathrm{eV}$, although the initial degree of spin polarization increases towards the absorption edge.

Above the absorption edge, $P_{S}$ first decreases and then reaches a secondary local maximum around $h v=2.3 \mathrm{eV}$. In fact, this energy nearly corresponds to transitions at the $L$ point of the BZ, where the crystal field reduces the symmetry and a splitting between heavy holes and light holes takes place. As a result, in a relatively narrow spectral region, it is possible to achieve very high spin polarization within the $L$ valleys. Unfortunately, with such a photon energy the generation of carriers is not restricted only to the $L$ valleys but involves several points of the BZ, so that the net degree of spin polarization reduces to $P_{S} \sim 20 \%$ [13]. However, at $2.3 \mathrm{eV}$, the absorption length is reduced by two orders of magnitude with respect to the case of $0.8 \mathrm{eV}$; in fact, there is a large region in the $k$ space where the $L_{6}$ conduction and $L_{4,5}$ valence bands are parallel, with effective masses larger than those at the $\Gamma$ point. The absorption at this energy is very effective so that $\lambda_{L} \ll l_{\mathrm{SF}}$ and we definitely enter the spin-orientation regime. It is thus clear why at $2.3 \mathrm{eV}$ we observe the absolute maximum of $l_{\mathrm{SF}}$ : the lower $P_{S}(20 \%)$ with respect to the value at $0.8 \mathrm{eV}(50 \%)$ is largely compensated by the much lower depolarization of photogenerated carriers during the propagation towards the 
barrier. These results unambiguously demonstrate the presence of a second peak at $530 \mathrm{~nm}$ in the degree of spin optical orientation, as theoretically predicted, and provide a solid framework for interpreting the spectral response of spin PDs.

We can gain a deeper insight into the complex spin relaxation phenomena occurring after optical spin pumping, by looking at the equivalent spin diffusion lengths used to fit our data with the diffusive model presented above. ${ }^{1}$ For $\lambda_{L}$ and $P_{S}$, we used the values reported in Fig. 3(b), while $l_{\mathrm{SF}}$ is left as a free parameter. The red and blue lines plotted in Fig. 3(a) represent the best fits to the experimental data in the 0.8 to $1.5 \mathrm{eV}$ photon energy range. A nice fitting can be obtained using constant value of $l_{\mathrm{SF}}$ equal to $0.9 \pm 0.1 \mu \mathrm{m}$ and $190 \pm 30 \mathrm{~nm}$ for electrons and holes, respectively, which are suitable values for our slightly doped $n$-Ge substrate and excitation close to the direct gap [9]. Assuming that the diffusive constant $D$ is $0.01035 \mathrm{~m}^{2} \mathrm{~s}^{-1}$ for electrons and $0.0049 \mathrm{~m}^{2} \mathrm{~s}^{-1}$ for holes [20], this corresponds to $\tau_{\mathrm{SF}}^{e l, \Gamma}=70 \pm 20 \mathrm{ps}$ for electrons and $\tau_{\mathrm{SF}}^{h, \Gamma}=7.5 \pm 2.5 \mathrm{ps}$ for holes. Nevertheless, the extension of the fit with the same parameters above $1.5 \mathrm{eV}$ photon energy [dashed lines in Fig. 3(b)] fails in reproducing experimental data, especially at $2.3 \mathrm{eV}$, where a much larger (lower) $A_{\mathrm{SF}}$ would be expected for holes (electrons). Indeed, a reasonable fit of our experimental data can be achieved here [continuous curves in the yellow area of Fig. 3(b)] only assuming that the equivalent spin lifetime for electrons is longer $\left(\tau_{\mathrm{SF}}^{e l, L}=155 \pm 35 \mathrm{ps}\right)$, while the one of holes shorter $\left(\tau_{\mathrm{SF}}^{h, L}=2.5 \pm 1.0 \mathrm{ps}\right)$. We will not discuss here the absolute values of relaxation times, because they arise from the interpretation of a complex phenomenon within a diffusive model and not from direct spectroscopic measurements. However, the trend is definitely interesting. The energy dependence of the spin relaxation time can be rationalized considering that the photon energy $h v$ also determines the point of the BZ where the photogeneration process takes place. Consider first the case of electrons. For $h v$ resonant with the direct band gap, electrons are primarily photogenerated close to the $\Gamma$ point of the BZ [see Fig. 1(a)] and then they relax with a characteristic time ( $\tau_{\Gamma \rightarrow L} \sim 230$ fs [18]) towards the CBM located at the $L$ point. This process takes place via an intervalley $\Gamma \rightarrow X \rightarrow L$

${ }^{1}$ For the fitting, we simply used the function $f(h v)$, as the second term within brackets of Eq. (2) does not contain any sizable dependence on the photon energy. phonon-assisted momentum scattering, as the $\Gamma X$ and $X L$ scattering rates are much higher than the $\Gamma L$ one [21]. We suggest that this process could strongly depolarize the carriers by efficient spin-flip relaxation due to the spin hotspot on the square face of the BZ, whose center is in the $X$ point, as pointed out for the case of $\mathrm{Si}$ [22]. For higher photon energies $(\sim 2.3 \mathrm{eV})$, the photogeneration process takes place at the $L$ point of the BZ. Electrons are already at the bottom of the conduction band, so that the spin depolarization phenomena are expected to be much less efficient [23]. For holes the situation is reversed, as they stay in $\Gamma$ for $h v$ resonant with the direct band gap and decay from $L$ to $\Gamma$ via the $X$ point when $h v \sim 2.3 \mathrm{eV}$. By consequence, the hole spin diffusion length decreases when increasing $h v$.

To summarize, our data provide the experimental proof of theoretical predictions for the spectral dependence of $P_{S}$ in Ge over a broad spectral range. We proved that, apart from the maximum in the spin polarization of photocarriers for photon energy resonant with the direct gap $(1550 \mathrm{~nm}$, $0.8 \mathrm{eV})$, there is a second sizable peak at $530 \mathrm{~nm}(2.3 \mathrm{eV})$ due to photogeneration in the $L$ valleys, far away from the center of the Brillouin zone. Noteworthy, the efficiency of conversion between light helicity and photocurrent modulation $\left(A_{\mathrm{SF}}\right)$ is higher at $530 \mathrm{~nm}$ than at $0.8 \mathrm{eV}$, due to the lower spin depolarization of carriers excited very close to the barrier at $530 \mathrm{~nm}$. Our data demonstrate that there exist different spin depolarization mechanisms for carriers excited around $\Gamma$ and $L$, suggesting lower spin relaxation times in case transitions through the $X$ point are involved. This work demonstrates that Ge-based spin PDs can be used as suitable integrated detectors of the photon helicity in a wide spectral range spanning from the infrared $(0.8 \mathrm{eV})$ to the visible $(2.3 \mathrm{eV})$. This paves the way to their use in several applications, not only related to telecom but also to other fields (e.g., biology), where the detection of the light helicity in the visible range could be of high interest.

The authors thank H. Dery, G. Isella, S. Sanvito, N. M. Caffrey, and D. Petti for fruitful discussions, R. Ward for the calculated Ge band structure, A. Melloni for help with the optical measurements, and M. Leone for his skillful technical support. This work was partially funded by Fondazione Cariplo, Grant No. 2013-0623, and by the project FIRB OSSIDI NANOSTRUTTURATI: MULTIFUNZIONALITÀ E APPLICAZIONI (RBAP115AYN).
[1] S. D. Ganichev, S. N. Danilov, V. V. Bel'kov, S. Giglberger, S. A. Tarasenko, E. L. Ivchenko, D. Weiss, W. Jantsch, F. Schäffler, D. Gruber, and W. Prettl, Phys. Rev. B 75, 155317 (2007).

[2] C. Shen, T. Trypiniotis, K. Y. Lee, S. N. Holmes, R. Mansell, M. Husain, V. Shah, X. V. Li, H. Kurebayashi, I. Farrer, C. H. de Groot, D. R. Leadley, G. Bell, E. H. C. Parker, T. Whall, D. A. Ritchie, and C. H. W. Barnes, Appl. Phys. Lett. 97, 162104 (2010).

[3] J.-M. Tang, B. T. Collins, and M. E. Flatté, Phys. Rev. B 85, 045202 (2012).
[4] E. J. Loren, J. Rioux, C. Lange, J. E. Sipe, H. M. van Driel, and A. L. Smirl, Phys. Rev. B 84, 214307 (2011).

[5] F. Bottegoni, G. Isella, S. Cecchi, and F. Ciccacci, Appl. Phys. Lett. 98, 242107 (2011).

[6] F. Bottegoni, A. Ferrari, G. Isella, S. Cecchi, M. Marcon, D. Chrastina, G. Trezzi, and F. Ciccacci, J. Appl. Phys. 111, 063916 (2012).

[7] F. Pezzoli, F. Bottegoni, D. Trivedi, F. Ciccacci, A. Giorgioni, P. Li, S. Cecchi, E. Grilli, Y. Song, M. Guzzi, H. Dery, and G. Isella, Phys. Rev. Lett. 108, 156603 (2012). 
[8] E.-S. Liu, J. Nah, K. M. Varahramyan, and E. Tutuc, Nano Letters 10, 3297 (2010).

[9] C. Rinaldi, M. Cantoni, D. Petti, A. Sottocorno, M. Leone, N. M. Caffrey, S. Sanvito, and R. Bertacco, Adv. Mater. 24, 3037 (2012).

[10] C. Rinaldi, M. Cantoni, D. Petti, and R. Bertacco, J. Appl. Phys. 111, 07C312 (2012).

[11] D. Petti, M. Cantoni, C. Rinaldi, S. Brivio, R. Bertacco, J. Gazquez, and M. Varela, J. Appl. Phys. 109, 084909 (2011).

[12] M. Cantoni, D. Petti, C. Rinaldi, and R. Bertacco, Appl. Phys. Lett. 98, 032104 (2011).

[13] J. Rioux and J. E. Sipe, Phys. Rev. B 81, 155215 (2010).

[14] T. T. Zhang, P. Barate, C. T. Nguyen, A. Balocchi, T. Amand, P. Renucci, H. Carrere, B. Urbaszek, and X. Marie, Phys. Rev. B 87, 041201 (2013).

[15] M. Cantoni, D. Petti, C. Rinaldi, and R. Bertacco, Microelectron. Eng. 88, 530 (2011).
[16] See Supplemental Material at http://link.aps.org/supplemental/ 10.1103/PhysRevB.90.161304 for information concerning the subtraction of the MCD contribution from the helicitydependent photocurrent, the transit time of carriers and the modified Fert-Jaffrès model used to calculate the spin transport asymmetry.

[17] S. Sze and K. Ng, Physics of Semiconductor Devices (John Wiley \& Sons, Inc., Hoboken, New Jersey, 2006).

[18] G. Mak and H. M. van Driel, Phys. Rev. B 49, 16817 (1994).

[19] W. C. Dash and R. Newman, Phys. Rev. 99, 1151 (1955).

[20] O. A. Golikova, B. Ya. Moizhez, and L. S. Stilbans, Sov. Phys. Solid State 3(10), 2259 (1962).

[21] F. Pezzoli, L. Qing, A. Giorgioni, G. Isella, E. Grilli, M. Guzzi, and H. Dery, Phys. Rev. B 88, 045204 (2013).

[22] P. Li and H. Dery, Phys. Rev. Lett. 107, 107203 (2011).

[23] P. Li, Y. Song, and H. Dery, Phys. Rev. B 86, 085202 (2012). 